\title{
Ventricular septal intramyocardial dissection after cardiac fibrosarcoma excision
}

\author{
Gang Wang ${ }^{1}$, Jianhua Wang ${ }^{2}$, Gengxu Zhou ${ }^{1}$, and Zhichun Feng ${ }^{1}$ \\ ${ }^{1}$ Southern Medical University \\ ${ }^{2}$ Chinese PLA General Hospital
}

October 30, 2021

\begin{abstract}
Intramyocardial dissection following cardiac tumor excision is uncommon. The evidence available is limited to few case reports.

${ }^{1}$ Herein,we report an infant with large cardiac fibrosarcoma arising from the interventricular septum and underwent surgical excision. One month after surgery echocardiography revealed a cystic dissection located in the interventricular septum with residual shunts within the ventricular chambers. we performed conservative strategy due to the high surgical risk, difficulty of interventional therapy and hemodynamic stability.
\end{abstract}

Ventricular septal intramyocardial dissection after cardiac fibrosarcoma excision Gang Wang $\mathrm{MM}^{1,2,+}$, Jianhua Wang $\mathrm{MD}^{3,+}$,Gengxu Zhou MD ${ }^{1,2}$,Zhichun Feng MD ${ }^{1,2, *}$

${ }^{1}$ Second Clinical Medical College,Southern Medical University,Guangzhou,China.

${ }^{2}$ Department of Pediatrics,The Seventh Medical Center of the PLA General Hospital, Beijing,China.

${ }^{3}$ Department of Ultrasound,The Seventh Medical Center of the PLA General Hospital, Beijing,China.

+ Gang Wang and Jianhua Wang are co-first authors on this work

${ }^{*}$ Correspondence:Zhichun Feng,Department of Pediatrics, The Seventh Medical Center of the PLA General Hospital,Beijing,100700,China;Second Clinical Medical College,Southern Medical University,Guangzhou,510515, China.

E-mail:fengzc111@126.com

\section{Abstract}

Intramyocardial dissection following cardiac tumor excision is uncommon. The evidence available is limited to few case reports. ${ }^{1}$ Herein,we report an infant with large cardiac fibrosarcoma arising from the interventricular septum and underwent surgical excision. One month after surgery echocardiography revealed a cystic dissection located in the interventricular septum with residual shunts within the ventricular chambers. we performed conservative strategy due to the high surgical risk, difficulty of interventional therapy and hemodynamic stability.

Key words : Intramyocardial dissection,Cardiac tumor

\section{Case presentation}

A 3-month-old female infant presented to our hospital with one month history of dyspnea.Transthoracic echocardiography demonstrated a large tumor mass $(50 \mathrm{~mm} \times 42 \mathrm{~mm})$ originated from the interventricular septum and filled the bilateral ventricular chambers(Figure 1). The patient underwent surgical resection of the 
tumor via the median sternotomy under cardiopulmonary bypass(CPB). During the operation, we found the tumor infiltrated the myocardium and the border between the tumor and the myocardium was unclear. Eventually we completely resected the tumor including part of myocardium. Interventricular septum was repaired with a bovine pericardium patch. The patient was weaned from the CPB and a postoperative transthoracic echocardiography confirmed no residual tumor and good ventricular function. Pathological examination revealed fibrosarcoma.

At one month follow up transthoracic echocardiography revealed a cystic dissection $(43 \mathrm{mmx} 32 \mathrm{~mm})$ located in the interventricular septum(Figure 2).This cystic dissection connected to the left ventricle through a $4.5 \mathrm{~mm}$ defect,and to the right ventricle through several small holes of its thin walls(Figure 3,Movie S1).We defined this case as ventricular septal intramyocardial cystic dissection with residual shunts within the ventricular chambers due to the separation of myocardial layers and communication with ventricular chambers after operation. we performed conservative strategy, because of the high surgical risk, difficulty of interventional therapy and hemodynamic stability. At 9 months of follow-up, transthoracic echocardiography demonstrated the original cystic dissection was slightly reduced and the shunts between the cystic dissection and right ventricle disappeared. There was bidirectional shunt between the cystic dissection and left ventricle through the defect(Figure 4,Movie S2). The patient was asymptomatic and cardiac function was good. We continue to follow up the patient.

\section{Discussion}

Intramyocardial dissection is rare and mostly secondary to myocardial infarction.In a meta-analysis ${ }^{2}$ of the literature including 40 cases of Intramyocardial dissection,32 cases after myocardial infarction and in 8 patients following trauma. ${ }^{3-4}$ In 5 patients, communication between the left and right ventricles was found. In-hospital mortality was $23 \%$. Multivariate analysis showed that the strongest independent predictor of mortality was $\mathrm{EF}<35 \% .{ }^{2}$ There also were a few reports of intramyocardial dissection secondary to the cardiac echinococcosis. ${ }^{5-6}$ Intramyocardial dissection following cardiac tumor excision is uncommon,the evidence available is limited to few case reports. This complication is due to destruction of the integrity of the interventricular septum and entry into the ventricular cavities during resection of the tumor. In our patient, only very thin endocardium separated the tumor from the ventricular cavity, entering into the left ventricular cavity when the tumor was dissected.we repaired the septum with a bovine pericardium patch. It may be due to the fragility of the myocardial tissue leading to the suture avulsion, followed by the formation of ventricular septal intramyocardial dissection and residual shunt.

The optimal management of intramyocardial dissection is controversial due to its rarity and lack of supportive evidence. Most of the experience come from studies of intramyocardial dissection after myocardial infarction. Treatment includes surgery, intervention and conservative medical therapy. In a systematic review of patients with postinfarction intramyocardial dissection, the mortality rate was significantly higher in the medical group versus surgical-treated group ( $85.7 \%$ versus $42.3 \%) .{ }^{7}$ For our patient, the age was only 3 months, interventional treatment was difficult, and the risk of reoperation was high. Most importantly, the patient was asymptomatic and hemodynamically stable.So we performed conservative strategy. During follow-up, we found that the cystic dissection was slightly reduced and the shunts between the cystic dissection and right ventricle disappeared. We will continue to follow up the patient.

Echocardiography is a noninvasive and simple technique.It has a important role in diagnosing intramyocardial dissection. Echocardiography

can evaluate the size and location of the intramyocardial dissection, detect flow within the cavity,assess heart function, thus assisting in the decisions about treatment strategy.We can learn from our case that when resecting tumors located in the ventricular septum, we should try to ensure the integrity of the ventricular septum and avoid entering the ventricular cavity. After resection, we should carefully check and repair the ventricular septum. For benign cardiac tumors can be partial resection to prevent damage to important structures. ${ }^{8}$

\section{References}


1. Wenjuan Bai,Lijun Zeng,Li Rao.A residual intramyocardial cystic dissection after cardiac fibroma resection.Echocardiography.2015;32:602-603.

2. Marina Leitman,Vladimir Tyomkin,Leonid Sternik,et al.Intramyocardial dissecting hematoma: Two case reports and a meta-analysis of the literature.Echocardiography.2018;00:1-7.

3. Maselli D,Micalizzi E,Pizio R,et al.Posttraumatic left ventricular pseudoaneurysm due to intramyocardial dissecting hematoma. Ann Thorac Surg.1997;64:830-831.

4. Silverstein JR,Tasset MR,Dowling RD,et al.Traumatic intramyocardial left ventricular dissection: a case report. J Am Soc Echocardiogr. 2006;19:1529. e5-8.

5. Ahmed T,Al-Zaibag M,Allan A,et al.Cardiac echinococcosis causing acute dissection of the left ventricular free wall. Echocardiography.2002;19:333-336.

6. Unal M, Tuncer C, Serce K, et al.A cardiac giant hydatid cyst of the interventricular septum masquerading as ischemic heart disease: Role of MR imaging. Acta Cardiol.1995;50:323-326.

7. Shokoufeh $\mathrm{H}, \mathrm{Abdollah} \mathrm{A}$,Marziyeh $\mathrm{P}$,et al.Postinfarction intramyocardial dissection, an interesting case report and systematic review. Echocardiography. 2019;00:1-8.

8. Padalino MA, Vida VL, Boccuzzo G, et al. Surgery for primary cardiac tumors in children: early and late results in a multicenter European Congenital Heart Surgeons Association study. Circulation. 2012;126:22-30.

\section{Hosted file}

figures.docx available at https://authorea.com/users/443739/articles/543679-ventricularseptal-intramyocardial-dissection-after-cardiac-fibrosarcoma-excision 